\title{
Biologia e exigências térmicas de Trichogramma pretiosum Riley (Hymenoptera: Trichogrammatidae) sobre ovos de Bonagota cranaodes (Meyrick) (Lepidoptera: Tortricidae)
}

\author{
Biology and thermal requirements of Trichogramma pretiosum Riley (Hymenoptera: \\ Trichogrammatidae) on eggs of Bonagota cranaodes (Meyrick) \\ (Lepidoptera: Tortricidae)
}

\author{
Regina da Silva Borba ${ }^{1}$ Mauro Silveira Garcia ${ }^{1}$ Adalécio Kovaleski ${ }^{2}$ \\ Andressa Comiotto $^{3}$ Renata Loureiro Cardoso ${ }^{1}$
}

\section{RESUMO}

\begin{abstract}
Este trabalho teve como objetivo estudar a biologia de Trichogramma em laboratório, visando a sua utilização em programas de manejo integrado para o controle de Bonagota cranaodes na cultura da macieira. A biologia de Trichogramma bruni Nagaraja e de duas linhagens $\left(L_{3}\right.$ e $\left.L_{4}\right)$ de Trichogramma pretiosum foi realizada sobre ovos de $\boldsymbol{B}$. cranaodes. Além disso, suas exigências térmicas e o número anual de gerações também foram determinados. A linhagem $L_{3}$ apresentou maior parasitismo (52,3\%), no entanto, a viabilidade foi semelhante entre as linhagens estudadas, com ciclo evolutivo de 8,7 dias, longevidade de fêmeas de 10,65 dias, temperatura base de $11,35^{\circ} \mathrm{C}$ e constante térmica de 128,70GD. O número de gerações anuais dessa linhagem em laboratório foi de 47,22 e de 13,59 gerações para as condições da região de Vacaria - RS. O baixo parasitismo de T. bruni e da linhagem $L_{4}$ de $T$. pretiosum pode estar ligado à camada de secreção existente sobre as posturas da praga. Embora este parasitismo seja considerado baixo, a utilização destas linhagens não pode ser descartada dentro do sistema de Produção Integrada da Maçã, devendo novos estudos serem realizados em condições de campo.
\end{abstract}

Palavras-chave: parasitóide de ovos, percentagem de parasitismo, controle biológico, Trichogramma, constante térmica.

\section{ABSTRACT}

This work was aimed at studyng the biology of Trichogramma in laboratory, in search of its use in integrated management programs to control Bonagota cranaodes in culture of the apple. The biology of Trichogramma bruni Nagaraja and of two lineages $\left(L_{3}\right.$ and $\left.L_{4}\right)$ of Trichogramma pretiosum on eggs of $\boldsymbol{B}$. cranaodes was done such their thermal demands and annual number of generations. The line $L_{3}$ presented more parasitism (52.3\%), however, the feseability was similar among the lineages studied; with biological cycle of 8.7 days; the longevity of females was 10.65 days; low threshold temperature of $11.35^{\circ} \mathrm{C}$ and thermal requirement of $128.70 \mathrm{GD}$, the number of annual generations this line in laboratory was 47.22 and 13.59 for Vacaria-RS climate conditions. The low parasitism of the T. bruni and of the lineage $L_{4}$ of $T$. pretiosum can be related to the layer of secretion presented on the laying of the pest. Although this parasitism is considered low, the use of these lineages cannot be discarded the Integrated Apple Production, systens. New studies shal be accomplished in field conditions.

Key words: parasitoid of the eggs, parasitism percentage, biological control, Trichogramma, thermal requirement.

\section{INTRODUÇÃO}

A lagarta-enroladeira Bonagota cranaodes (Meyrick) (Lepidoptera: Tortricidae) tem-se tornado, nos últimos anos, uma das principais pragas da cultura da macieira no Brasil. Em duas das principais regiões produtoras (Fraiburgo, SC e Vacaria, RS), anualmente são realizadas até oito pulverizações com inseticidas fosforados visando ao controle desta praga; no entanto, as perdas na produção têm-se situado entre 3 e 5\%. O grande número de aplicações deve-se, possivelmente, ao hábito do inseto de se proteger no interior da folhagem, o que reduz a eficiência dos inseticidas (BOTTON et al., 2000).

\footnotetext{
${ }^{1}$ Departamento de Fitossanidade, Universidade Federal de Pelotas (UFPel). CP 354, 96010-970, Pelotas, RS, Brasil. E-mail: rsborba@pop.com.br. Autor para correspodência.

${ }^{2}$ Centro Nacional de Pesquisa de Uva e Vinho, Empresa Brasileira de Pesquisa Agropecuária (Embrapa), Bento Gonçalves, RS, Brasil.

${ }^{3}$ Instituto de Biologia, UFPel, Pelotas, RS, Brasil.
} 
A baixa eficiência do controle químico, aliada ao desequilíbrio causado pela aplicação de produtos químicos de amplo espectro de ação, torna fundamental a busca de alternativas de controle desta praga.

Uma alternativa para o uso de inseticidas no controle da praga é o emprego de inimigos naturais, como os microhimenópteros do gênero Trichogramma, que se apresentam como uma opção que pode ser muito promissora. Esses insetos parasitam ovos de inúmeras pragas agrícolas e florestais, principalmente da ordem Lepidoptera (SMITH, 1996; HASSAN, 1997). Um dos motivos do sucesso de parasitóides no controle biológico é que, além de atacar o estágio inicial de desenvolvimento das pragas, apresenta facilidade de criação (PARRA et al., 1989).

Espécies do gênero Trichogramma controlam as pragas na fase de ovo, antes destas causarem qualquer dano à cultura, sendo uma das principais vantagens do emprego dessas espécies.

As lagartas de $\boldsymbol{B}$. cranaodes abrigam-se entre as folhas, entre os frutos ou na região do cálice ou do pedúnculo (KOVALESKI, 1996), o que torna difícil atingi-las com pulverizações de inseticidas, resultando na baixa eficiência desses produtos, aumentando a importância de se controlar a praga antes que ela se abrigue nestes locais.

Para se ter sucesso em um programa de controle biológico com Trichogramma, é necessário selecionar a espécie e a linhagem do parasitóide que controle mais eficientemente a praga e que melhor se adapte à cultura e às condições climáticas da região onde será utilizado. O uso de uma linhagem local é preferido em relação a uma importada porque esta é mais adaptada ao ambiente da região (HASSAN, 1997). Assim, este trabalho teve como objetivo realizar a biologia de duas linhagens de $\boldsymbol{T}$. pretiosum e uma de $\boldsymbol{T}$. bruni sobre ovos de $\boldsymbol{B}$. cranaodes, bem como determinar as exigências térmicas e a estimativa do número de gerações anuais para as condições climáticas de origem para as linhagens de T. pretiosum.

\section{MATERIAL E MÉTODOS}

Os ovos de B. cranaodes foram obtidos na criação de manutenção do Laboratório de Biologia de Insetos do Departamento de Fitossanidade da Faculdade de Agronomia “Eliseu Maciel” (FAEM), da Universidade Federal de Pelotas (UFPel). O trabalho foi conduzido utilizando as linhagens $\mathrm{L}_{3}$ e $\mathrm{L}_{4}$ de $\boldsymbol{T}$. pretiosum, coletadas em pomares de macieira no município de Vacaria - RS, e a linhagem $\mathrm{L}_{9}$ de $\boldsymbol{T}$. bruni, oriunda da coleção da ESALQ/Piracicaba-SP.
Para a determinação da biologia, à medida que os adultos de Trichogramma das duas espécies emergiam $\left(\mathrm{L}_{3}, \mathrm{~L}_{4}\right.$ e $\left.\mathrm{L}_{9}\right)$, os tubos eram fechados com filme plástico de PVC $\left(\right.$ Magipack $^{\circledR}$ ), colocando-se um pedaço de algodão umedecido com solução aquosa de mel a 50\% para alimentação dos parasitóides. A seguir, em cada tubo foram colocadas posturas de $\boldsymbol{B}$. cranaodes com idade inferior a $24 \mathrm{~h}$ na proporção de 1 fêmea do parasitóide para 10 ovos da praga, por um período de parasitismo de 24 horas. O mesmo procedimento foi realizado utilizando ovos do hospedeiro alternativo Anagasta kuehniella (Zeller, 1879) (Lepidoptera: Pyralidae). Os ovos foram incubados em câmara climatizada a temperatura de $25 \pm 1^{\circ} \mathrm{C}$, umidade relativa de $70 \pm 12 \%$ e fotofase de 14 horas, com 20 repetições para cada linhagem. Os parâmetros avaliados foram a percentagem de parasitismo, a viabilidade, a duração do período ovoadulto, a razão sexual e o número de parasitóides por ovo. O sexo dos indivíduos foi determinado através do dimorfismo apresentando pelas antenas, com o auxílio de um microscópio esteroscópico.

Foi realizada a determinação da temperatura base para as duas linhagens de T. pretiosum $\left(\mathrm{L}_{3}\right.$ e $\left.\mathrm{L}_{4}\right)$, nas quais foi incubada uma postura contendo em torno de 20 ovos de $\boldsymbol{B}$. cranaodes, recém-parasitados, em câmaras climatizadas do tipo BOD, reguladas nas temperaturas de $14,18,22,25$ e $28^{\circ} \mathrm{C}$, com umidade relativa de $70 \pm 12 \%$ e fotofase de 14 horas, sendo utilizadas 20 repetições por temperatura. Os parâmetros avaliados foram os mesmos citados anteriormente.

Para o cálculo do limiar inferior (Tb) e da constante térmica $(\mathrm{K})$, foi utilizado o método da hipérbole (HADDAD et al., 1999), baseando-se na duração do período de ovo-adulto, obtido nas diferentes temperaturas, por meio do Programa MOBAE (Modelo bioestatístico aplicado à entomologia). $\mathrm{O}$ delineamento experimental foi inteiramente casualizado, com 20 repetições. Como os parâmetros avaliados para as diferentes temperaturas são quantitativos, foram realizadas análises de regressão. As análises estatísticas foram realizadas pelo programa "Genes”, e as médias foram comparadas pelo teste de Tukey em nível de 5\% de probabilidade de erro.

Foi calculado o número de gerações anuais em laboratório, na temperatura constante de $28^{\circ} \mathrm{C}$, e, para a estimativa em condições de campo, foram utilizadas as normais térmicas dos últimos 20 anos (19832002) do município de Vacaria - RS, fornecidas pela estação meteorológica da Embrapa Vacaria. 


\section{RESULTADOS E DISCUSSÃO}

Em relação à agressividade de Trichogramma em ovos de $\boldsymbol{B}$. cranaodes, não houve diferença estatística entre as linhagens, porém a linhagem $L_{3}$ de $T$. pretiosum apresentou um parasitismo de 52,3\% (Tabela 1). O mesmo ocorreu no trabalho de PRATISSOLI (1995), no qual não foram observadas diferenças estatísticas com relação ao parasitismo de ovos de Tuta absoluta (Meirick, 1917) (Lepidoptera: Gelechiidae), entre as seis linhagens de T. pretiosum estudadas, apresentando valores de parasitismo que variaram de 43,46 a 69,47\%. Já em ovos de Phthorimaea operculella (Zeller, 1873) (Lepidoptera: Gelechiidae), três linhagens se mostraram mais agressivas, apresentando valores de parasitismo em torno de $70 \%$.

A percentagem de parasitismo das três linhagens de Trichogramma sobre ovos do hospedeiro alternativo A. kuehniella foi superior em relação aos ovos de $\boldsymbol{B}$. cranaodes. Isto pode estar relacionado ao efeito pré-imaginal (KAISER et al., 1989), já que estes tricogramatídeos vinham sendo multiplicados em ovos de A. kuehniella. Outro fator que pode estar relacionado ao baixo parasitismo é a camada de secreção existente nas posturas de $\boldsymbol{B}$. cranaodes, fato também relatado por BASSO et al. (1998); HONDA \& LUCK(2000); FONSECA(2001). Este último, ao estudar a biologia de quatro linhagens de $\boldsymbol{T}$. pretiosum também em ovos de $\boldsymbol{B}$. cranaodes, observou índices de parasitismo menores que os obtidos no presente trabalho, no qual a percentagem de parasitismo variou de 10,62 a 25,65\%. BASSO et al. (1998) trabalharam com duas linhagens de T. pretiosum sobre ovos de B. cranaodes e Argyrotaenia sphaleropa (Meyrick) (Lepidoptera: Tortricidae) e observaram que este parasitóide possui preferência por ovos de $\boldsymbol{A}$. sphaleropa e que ovos de $\boldsymbol{B}$. cranaodes não seriam adequados, apesar de serem parasitados no campo. A não-preferência por ovos de B. cranaodes pode estar ligada à camada de secreção transparente que cobre as posturas, provavelmente, utilizada como obstáculo ao parasitismo. HONDA \& LUCK (2000), estudando a aceitabilidade de ovos de Amorbia cuneana (Walsingham) (Lepidoptera: Tortricidae) e Sabulodes aegrotata (Guenée) (Lepidoptera: Geometridae) por T. platneri Nagarkatti, concluíram que o córion espesso de $\boldsymbol{S}$. aegrotata e a camada pegajosa destes ovos causaram o baixo parasitismo. VINSON (1997) observou que a dureza e a espessura do córion são fatores que afetam a aceitação do hospedeiro.

Os valores de parasitismo encontrados neste trabalho foram inferiores aos de GARCIA (1998), ao estudar o potencial de parasitismo de T. pretiosum para controle biológico do bicho-furão-dos-citrus Ecdytolopha aurantiana (Lima) (Lepidoptera: Tortricidae), que encontrou um parasitismo de 81 a $100 \%$, ressaltando-se que a postura deste inseto é isolada e sem cobertura.

A viabilidade em ovos de $\boldsymbol{B}$. cranaodes variou de 61,7 a $68,3 \%$ e de 85,4 a $89,6 \%$ para $\boldsymbol{A}$. kuehniella, respectivamente (Tabela 1). Já no trabalho de FONSECA (2001), a viabilidade de quatro linhagens de $\mathbf{T}$. pretiosum sobre ovos do mesmo hospedeiro variou de 28,09 a 39,08\%, sendo que, segundo este autor, esta baixa viabilidade poderia ser atribuída a problemas ocorridos no momento da emergência. PRATISSOLI \& PARRA(2001) obtiveram valores altos de viabilidade quando estudaram a biologia de seis

Tabela 1 - Taxa de parasitismo $(\mathrm{X} \pm \mathrm{EP})$, de viabilidade $(\mathrm{X} \pm \mathrm{EP})$, de duração do ciclo $(\mathrm{X} \pm \mathrm{EP})$, de razão sexual e de número de indivíduos por ovo de Trichograma pretiosum (linhagens $\mathrm{L}_{3}$ e $\mathrm{L}_{4}$ ) e de Trichogramma bruni (linhagem $\mathrm{L}_{9}$ ) em ovos de Bonagota cranaodes e do hospedeiro alternativo Anagasta kuehniella. Temperatura $25 \pm 1^{\circ} \mathrm{C}$, UR de $70 \pm 12 \%$ e fotofase de 14 horas.

\begin{tabular}{|c|c|c|c|c|c|c|}
\hline Hospedeiros & Linhagem & Parasitismo(\%) & Viabilidade(\%) & Duração(dias) & Razão sexual (\%) & $\mathrm{N}^{\circ}$ de indivíduos/ ovo \\
\hline \multirow{3}{*}{ B. cranaodes } & $\mathrm{L}_{3}$ & $52,3 \pm 6,72 a^{1}$ & $68,3 \pm 8,01$ a & $8,71 \pm 0,32 \mathrm{a}$ & $0,62 \mathrm{~b}$ & $1,15 \mathrm{a}$ \\
\hline & $\mathrm{L}_{4}$ & $45,3 \pm 4,97 \mathrm{a}$ & $61,7 \pm 4,63$ a & $8,50 \pm 0,16$ a & $0,54 \mathrm{~b}$ & $1,14 \mathrm{a}$ \\
\hline & $\mathrm{L}_{9}$ & $47,0 \pm 5,54$ a & $62,6 \pm 6,51$ a & $8,50 \pm 0,13$ a & $0,80 \mathrm{a}$ & $1,14 \mathrm{a}$ \\
\hline CV (\%) & & 52,4 & 44,14 & 11,53 & 36,74 & 47,32 \\
\hline \multirow{3}{*}{ A. kuehniella } & $\mathrm{L}_{3}$ & $86,2 \pm 2,89 a b$ & $85,4 \pm 5,60 \mathrm{a}$ & $7,47 \pm 0,06$ a & $0,49 \mathrm{~b}$ & 1,07 a \\
\hline & $\mathrm{L}_{4}$ & $78,6 \pm 4,11 \mathrm{~b}$ & $86,8 \pm 3,25$ a & $7,35 \pm 0,09$ a & $0,28 \mathrm{c}$ & $1,01 \mathrm{a}$ \\
\hline & $\mathrm{L}_{9}$ & $92,8 \pm 2,36$ a & $89,6 \pm 3,10$ a & $7,56+0,07$ a & $0,76 \mathrm{a}$ & $1,07 \mathrm{a}$ \\
\hline CV (\%) & & 16,36 & 21,20 & 4,49 & 37,63 & 12,32 \\
\hline
\end{tabular}

${ }^{1}$ Médias não seguidas pela mesma letra nas colunas, para cada hospedeiro, diferem estatisticamente entre si pelo teste de Tukey em nível de $5 \%$ de probabilidade de erro. 
linhagens de $\boldsymbol{T}$. pretiosum em ovos de $\boldsymbol{T}$. absoluta e $\boldsymbol{P}$. operculella, com valores variando de 82,6 a 98,3\% e de 83,9 a $98,9 \%$, respectivamente. Ressaltam que, no controle de qualidade da produção massal de Trichogramma, a viabilidade é considerada satisfatória quando o percentual de adultos emergidos for superior a $85 \%$.

Assim como a percentagem de parasitismo, os valores de viabilidade sobre o hospedeiro alternativo também foram altos, tendo o mesmo ocorrido no trabalho de BLEICHER \& PARRA (1989), quando estudaram três populações de Trichogramma em ovos de A. kuehniella, obtendo valores que variaram de 92 a $100 \%$. Resultados semelhantes também foram encontrados por HASSAN \& ZHANG (2001), estudando o parasitismo de $\boldsymbol{T}$. brassicae em ovos de Ostrinia nubilalis (Hübner) (Lepidoptera: Crambidae). HOFFMANN et al. (2000), estudando o desempenho de T. ostriniae em ovos de Sitotroga cerealella (Oliv.), e PRATISSOLI \& OLIVEIRA (1999), avaliando a influência da idade dos ovos de Helicoverpa zea (Bod., 1850) (Lepidoptera: Noctuidae) no parasitismo de $\boldsymbol{T}$. pretiosum, concluíram que a melhor taxa de emergência foi nos ovos com um dia de idade (91,63\%).

Não houve diferença significativa na duração do ciclo (ovo-adulto) entre as linhagens estudadas em ovos de $\boldsymbol{B}$. cranaodes, porém o ciclo se mostrou levemente superior ao encontrado em ovos de A. kuehniella (Tabela 1). Para as linhagens testadas por FONSECA (2001) em ovos do mesmo hospedeiro, a duração do ciclo foi maior que a apresentada neste trabalho. Isto também ocorreu nos trabalhos de PRATISSOLI (1995), em que o ciclo teve uma duração em torno de 10 dias, tanto para o hospedeiro T. absoluta como para P. operculella, e de BLEICHER (1985) que, testando três populações de Trichogramma em ovos de A. kuehniella, obteve valores que variaram de 9,73 a 11,08 dias.

A razão sexual em ovos de $\boldsymbol{B}$. cranaodes apresentou valores acima de 0,50 para as duas espécies de Trichogramma, sendo que, para a linhagem $\mathrm{L}_{9}$ de T. bruni, esses valores foram significativamente superiores comparados às demais linhagens, para ambos os hospedeiros. Este é um resultado desejável, pois indica um número maior de fêmeas em relação a machos, o que é importante para o controle biológico (Tabela 1). PRATISSOLI (1995) obteve resultados semelhantes quando estudou a biologia de $\mathbf{T}$. pretiosum em ovos de $\boldsymbol{P}$. operculella e T. absoluta, pois também observou um número maior de fêmeas. O mesmo ocorreu no trabalho de BLEICHER \& PARRA (1989), no qual, para duas populações de $T$. pretiosum sobre ovos de A. kuehniella, não houve presença de machos, e, para outra população, a razão sexual foi de 0,57 . No entanto, para FONSECA (2001), a razão sexual foi inferior a 0,50 em três das quatro linhagens estudadas de $\boldsymbol{T}$. pretiosum, também sobre ovos de $\boldsymbol{B}$. cranaodes. No presente trabalho, razão sexual abaixo de 0,5 foi observada nas duas linhagens de T. pretiosum em ovos de A. kuehniella (Tabela 1).

O número de parasitóides emergidos por ovo foi acima de 1 , tanto em ovos de $\boldsymbol{B}$. cranaodes como em ovos de A. kuehniella, não diferindo estatisticamente entre si (Tabela 1). Estes resultados estão próximos aos encontrados por BLEICHER \& PARRA(1989), PRATISSOLI (1995), PRATISSOLI \& OLIVEIRA(1999), ALENCAR et al. (2000); FONSECA (2001). Porém, valores inferiores ao encontrado nesta pesquisa foram observados por CÔNSOLI et al. (2000) que, estudando o parasitismo de duas espécies de Trichogramma, verificaram valores de 0,98 para $\boldsymbol{T}$. galloi Zucchi, 1988 (Hymenoptera: Trichogrammatidae) e de 0,84 para T. pretiosum, ambos em ovos do hospedeiro alternativo. Em relação a essas diferenças encontradas entre os autores, sobre este parâmetro, o tamanho do ovo é o principal responsável.

A duração do ciclo das linhagens $\mathrm{L}_{3}$ e $\mathrm{L}_{4}$ de T. pretiosum sobre ovos de $\boldsymbol{B}$. cranaodes foi afetada significativamente pela temperatura, ocorrendo um aumento na velocidade de desenvolvimento com a elevação térmica (Figura 1). O mesmo foi observado por BLEICHER \& PARRA (1989), concluindo que a duração do ciclo é inversamente proporcional ao aumento de temperatura.

As análises de regressão foram significativas para os parâmetros duração do ciclo evolutivo e viabilidade para as linhagens $\mathrm{L}_{3}$ e $\mathrm{L}_{4}$, mostrando respostas quadráticas e cúbicas. Comparando-se os valores dos coeficientes de determinação $\left(\mathrm{r}^{2}\right)$, pode-se constatar que os valores são muito próximos e sempre acima de 90\% (Figuras 1 e 2).

Em ambas as linhagens, a maior viabilidade foi obtida a $28^{\circ} \mathrm{C}(86,3 \%)$. Tanto para $\mathrm{L}_{3}$ como para $\mathrm{L}_{4}$, a melhor temperatura para desenvolvimento do parasitóide foi $28^{\circ} \mathrm{C}$ e a menos favorável foi $14^{\circ} \mathrm{C}$ (Figura 2), fato corroborado por AVANCI \& FOERSTER (2001) que, ao estudarem o efeito da temperatura no desenvolvimento de Trichogramma spp., concluíram que a percentagem de emergência de T. Iassalei Pinto foi mais suscetível à temperatura de $14^{\circ} \mathrm{C}$. Estes dados estão próximos aos de PRATISSOLI (1995), AVANCI \& FOERSTER (2001), PRATISSOLI \& PARRA (2001) e FONSECA(2001).

A razão sexual de ambas as linhagens de $\boldsymbol{T}$. pretiosum não apresentou diferença estatística, 

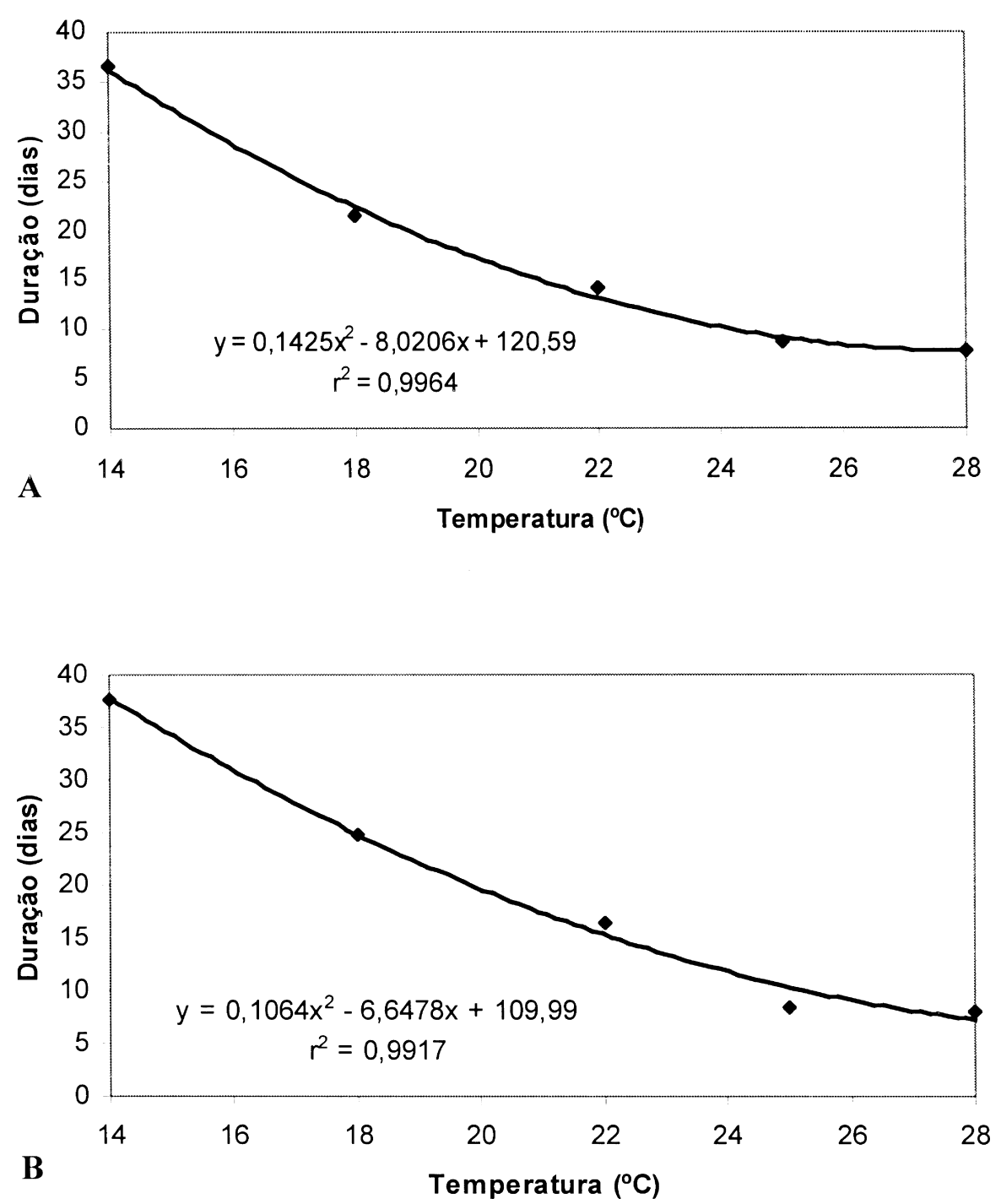

Figura 1 - Gráficos e equações de regressão e Coeficientes de determinação $\left(\mathrm{r}^{2}\right)$. A e B: Duração do ciclo evolutivo (dias) em diferentes temperaturas das Linhagens $\mathrm{L}_{3}$ e $\mathrm{L}_{4}$, respectivamente. UR de $70 \pm 12 \%$ e fotofase de 14 horas.

demonstrando que a temperatura não afetou esta variável, sendo os valores obtidos todos acima de 0,50 , com uma média de 0,58 para a Linhagem $\mathrm{L}_{3}$ e de 0,56 para $\mathrm{L}_{4}$. HANSEN \& JENSEN (2002), estudando o efeito da temperatura no parasitismo de T. turkestanica Meyer sobre ovos de Ephestia kuehniella Keller (Lepidoptera: Pyralidae), também encontraram resultados semelhantes de fêmeas em relação a machos.

Valores semelhantes aos encontrados neste trabalho foram relatados por PRATISSOLI (1995), que, ao determinar a razão sexual de $\boldsymbol{T}$. pretiosum sobre $\boldsymbol{T}$. absoluta, não observou interferência da temperatura neste parâmetro. No entanto, sobre ovos de $\boldsymbol{P}$. operculella, houve diferença estatística entre os valores de viabilidade nas diferentes temperaturas.

Para número de indivíduos emergidos por ovo de B. cranaodes, não ocorreu diferença estatística entre as temperaturas, para ambas as linhagens, sendo esta sempre superior a 1, com uma média de 1,10 indivíduos/ovo para ambas as linhagens. Resultados semelhantes foram relatados por BLEICHER (1985), ao estudar três populações de Trichogramma sobre ovos de A. kuehniella; PRATISSOLI (1995), estudando o parasitismo de T. pretiosum em ovos de T. absoluta e P. operculella; CÔNSOLI et al. (2000), estudando o parasitismo de T. galloi sobre ovos de A. kuehniella, 


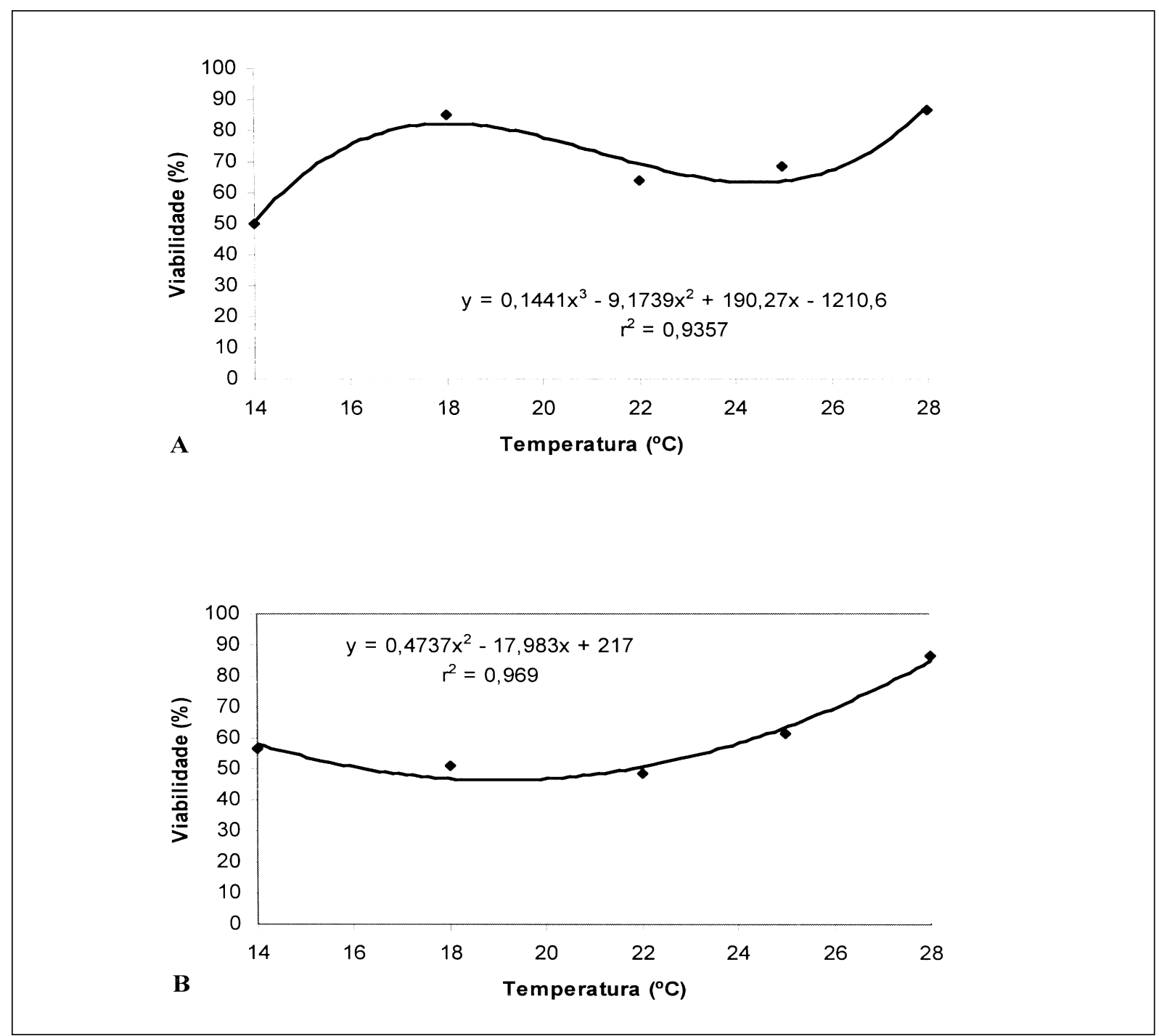

Figura 2 - Gráficos e equações de regressão e coeficientes de determinação $\left(\mathrm{r}^{2}\right)$. A e B: Viabilidade (\%) em diferentes temperaturas das Linhagens $\mathrm{L}_{3}$ e $\mathrm{L}_{4}$, respectivamente. UR de $70 \pm 12 \%$ e fotofase de 14 horas.

em diferentes temperaturas; e FONSECA (2001), ao estudar o efeito da temperatura sobre a biologia de $\boldsymbol{T}$. pretiosum sobre ovos de B. cranaodes.

Os valores da Temperatura Base (Tb), da constante térmica (K) e do coeficiente de determinação $\left(\mathrm{r}^{2}\right)$ para as duas linhagens de $\boldsymbol{T}$. pretiosum são apresentados na tabela 2.

As variações ocorridas na $\mathrm{Tb}$ e $\mathrm{K}$ estão diretamente relacionadas com a linhagem de Trichogramma e com o hospedeiro de criação (PRATISSOLI,1995; PRATISSOLI et al., 1998).

Os números de gerações anuais encontradas para as linhagens $\mathrm{L}_{3} \mathrm{e} \mathrm{L}_{4}$ foram de $47,22 \mathrm{e}$ de 45,51, respectivamente, para condições de laboratório, considerando-se a temperatura de $28^{\circ} \mathrm{C}$. Para as condições de campo, levando-se em consideração as normais térmicas para o município de Vacaria - RS, os números de gerações seriam de 13,59 e 12,42, respectivamente (Tabela 2).

As linhagens de Trichogramma estudadas neste trabalho não apresentaram índices de parasitismo muito satisfatórios. No entanto, para que se possa obter um controle desejado a campo (80\%), serão necessários novos estudos, no sentido de se estimar o número de ovos de B. cranaodes por hectare de pomar, assim como para determinar o raio de dispersão do parasitóide, não descartando, portanto, a utilização desses parasitóides dentro do sistema de Produção Integrada da Maçã.

Ciência Rural, v.36, n.5, set-out, 2006. 
Tabela 2 - Temperatura base (Tb), constante térmica (K), coeficiente de determinação ( $\mathrm{R}^{2}$ ) e estimativa do número de gerações de Trichogramma pretiosum (Linhagens $\mathrm{L}_{3}$ e $\mathrm{L}_{4}$ ) em ovos de Bonagota cranaodes, em Vacaria-RS e em laboratório. UR de $70 \pm 12 \%$ e fotofase de 14 horas.

\begin{tabular}{lccccc}
\hline Linhagem & $\mathrm{Tb}\left({ }^{\circ} \mathrm{C}\right)$ & $\mathrm{K}(\mathrm{GD})$ & $\left(\mathrm{R}^{2}\right)$ & $\mathrm{N}^{\mathrm{o}}$ de gerações(Vacaria-RS) & $\mathrm{N}^{\mathrm{o}}$ de gerações(laboratório) \\
\hline $\mathrm{L}_{3}$ & 11,35 & 128,70 & 96,30 & 13,59 & 47,22 \\
$\mathrm{~L}_{4}$ & 11,79 & 130,02 & 91,01 & 12,42 & 45,51 \\
\hline
\end{tabular}

\section{REFERÊNCIAS}

ALENCAR, J.A. de et al. Biologia de Trichogramma pretiosum Riley em ovos de Sitotroga cerealella (Olivier). Pesquisa Agropecuária Brasileira, Brasília, v.35, n.8, p.1669-1674, 2000.

AVANCI, M.R.F.; FOERSTER, L.A. Efeito da temperatura no desenvolvimento, reprodução e longevidade de cinco espécies de Trichogramma (Hymenoptera: Trichogrammatidae). In: SIMPÓSIO DE CONTROLE BIOLÓGICO, 7., 2001, Poços de Caldas, MG. Resumos... Poços de Caldas, 2001. p.375. CDROOM.

BASSO, C. et al. Comparacion de los caracteres biológicos y etológicos de Trichogramma pretiosum y de T. exiguum (Hymenoptera: Trichogrammatidae). Revista Chilena Entomológia, v.25, p.45-53, 1998.

BLEICHER, E. Biologia e exigências térmicas de populações de Trichogramma (Hymenoptera: Trichogrammatidae). 1985. 80f. Tese (Doutorado em Ciências) - Escola Superior de Agricultura "Luiz de Queiroz", Universidade de São Paulo.

BLEICHER, E.; PARRA, J.R.P. Espécies de Trichogramma parasitóides de Alabama argillacea. i. Biologia de três populações. Pesquisa Agropecuária Brasileira, Brasília, v.24, n.8, p.929-940, 1989.

BOTTON, M. et al. Exigências térmicas e estimativa do número de gerações de Bonagota cranaodes (Meyrick) (Lepidoptera: Tortricidae) em regiões produtoras de maçã do sul do Brasil. Anais da Sociedade Entomológica do Brasil, v.29, n.4, p.633-637, 2000.

CÔNSOLI, F.L. et al. Estimating parasitoid immature mortality by comparing oviposition and pupal development of Trichogramma galloi Zucchi and T. pretiosum Riley on natural and factitious hosts. Revista Brasileira de Biologia, v.60, n.3, p.381-387, 2000.

FONSECA, F.L. da. Ocorrência de Trichogramma (Hymenoptera: Trichogrammatidae) nas localidades de Pelotas, Bento Gonçalves e Vacaria, RS e aspectos bioecológicos em ovos de Bonagota cranaodes (Meyrick, 1937) (Lepidoptera: Tortricidae). 2001. 56f. Dissertação (Mestrado em Fitossanidade) - Faculdade de Agronomia "Eliseu Maciel”, Universidade Federal de Pelotas.

GARCIA, M.S. Bioecologia e potencial de controle biológico de Ecdytolopha aurantiana (Lima, 1927) (Lepidoptera: Tortricidae), o bicho-furão-dos-citros, através de Trichogramma pretiosum Riley, 1879. 1998. 118f. Tese (Doutorado em Ciências) - Escola Superior de Agricultura "Luiz de Queiroz”, Universidade de São Paulo.
HADDAD, M.L. et al. Métodos para estimar os limites térmicos inferior e superior de desenvolvimento de insetos. Piracicaba: FEALQ, 1999. 29p.

HANSEN, L.S.; JENSEN, K.M.V. Effect of temperature on parasitism and host-feeding of Trichogramma turkestanica (Hymenoptera: Trichogrammatidae) on Ephestia kuehniella (Lepidoptera: Pyralidae). Journal of Economic Entomology, v.95, n.1, p.50-56, 2002.

HASSAN, S.A. Seleção de espécies de Trichogramma para o uso em programas de controle biológico. In: PARRA, J.R.P.; ZUCCHI, R.A. Trichogramma e o controle biológico aplicado. Piracicaba: FEALQ, 1997. Cap.7, p.183-206.

HASSAN, S.A.; ZHANG, W.Q. Variability in quality of Trichogramma brassicae (Hymenoptera: Trichogrammatidae) from commercial suppliers in Germany. Biological Control, v.22, p.115-121, 2001.

HOFFMANN, M.P. et al. Performance of Trichogramma ostriniae (Hymenoptera: Trichogrammatidae) reared on factitious hosts, including the target host, Ostrinia nubilalis (Lepidoptera: Crambidae). Biological Control, v.21, p.110, 2000.

HONDA, J.Y.; LUCK, R.F. Age and suitability of Amorbia cuneana (Lepidoptera: Tortricidae) and Sabulodes aegrotata (Lepidoptera: Geometridae) eggs for Trichogramma platneri (Hymenoptera: Trichogrammatidae). Biological Control, v.18, p.79-85, 2000.

KAISER, L. et al. Behavioural study of plasticity in host preferences of Trichogramma maidis (Hym.: Trichogrammatidae). Physiological Entomology, v.14, p.1, p.53-60, 1989.

KOVALESKI, A. Manejo da lagarta enroladeira Phtheocroa cranaodes (Meyrick) em pomares de macieira. In: ENCONTRO DE FRUTICULTURA DE CLIMA TEMPERADO, 1., 1996, Vacaria. Anais... Caxias do Sul: UCS, 1996. p.42-43.

PARRA, J.R.P. et al. Número ideal de ovos de Anagasta kuehniella (Zeller, 1879) por caixa de criação para pesquisas com Trichogramma spp. Anais da Sociedade Entomológica do Brasil, v.18, p.391-402, 1989.

PRATISSOLI, D. Bioecologia de Trichogramma pretiosum Riley, 1978, nas traças Scrobipalpuloides absoluta e Phthorimaea operculella (Zeller, 1873), em tomateiro. 1995. 135f. Tese (Doutorado em Ciências) - Escola Superior de Agricultura “Luiz de Queiroz”, Universidade de São Paulo. 
PRATISSOLI, D. et al. Estimativa do número de gerações de Trichogramma pretiosum Riley na traça do tomateiro Tuta absoluta (Meyrick), com base nas exigências térmicas. Anais da Sociedade Entomológica do Brasil, v.27, n.1, p.109115, 1998.

PRATISSOLI, D.; OLIVEIRA, H.N. de. Influência da idade dos ovos de Helicoverpa zea (Boddie) no parasitismo de Trichogramma pretiosum Riley. Pesquisa Agropecuária Brasileira. Brasília, v.34, n.5, p.891-896, 1999.

PRATISSOLI, D.; PARRA, J.R.P. Seleção de linhagens de Trichogramma pretiosum Riley (Hymenoptera:
Trichogrammatidae) para o controle das traças Tuta absoluta (Meyrick) e Phthorimaea operculella (Zeller) (Lepidoptera: Gelechiidae). Neotropical Entomology, v.30, p.277-282, 2001

SMITH, S.M. Biological control with Trichogramma: advances, successes, and potential of their use. Annual Review of Entomology, v.41, p.375-406, 1996.

VINSON, S.B. Comportamento de seleção hospedeira de parasitóides de ovos, com ênfase na família Trichogrammatidae. In: PARRA, J.R.P.; ZUCCHI, R.A. Trichogramma e o controle biológico aplicado. Piracicaba: FEALQ, 1997. Cap.3, p.67119. 